\title{
Discussion to the papers by Henry T. Howat, A. V. Pollock and W. Sircus
}

\author{
Chairman: Dr J. B. LYNCH
}

LesLIE. Have we now disposed completely of the myth that chronic pancreatitis causes obstructive jaundice by cicatrization of the common duct. Would you agree that any jaundice which occurs is probably infective?

Howat. Oh no, certainly not, if you do cholangiograms you very often find the bile duct cut off at the top end of the pancreas indicating that obstruction is very important indeed in the production of jaundice.

HaNCOCK. Can you tell me about the lumen of the tubes you use in your pancreozymin and secretin tests. Some workers use two tubes and consider the lumen very important?

Howat. I am sorry, I can't tell you the exact lumen of the tubes, we use a radio-opaque duble lumen tube which is officially known as a Levine tube to which we attach a balloon to persuade it to pass into the duodenum.

QUESTION. Is that technique very difficult to perform?

Howat. The short answer to this is yes, it is difficult to perform if you are only doing it occasionally, but it is not difficult if you are doing two or three a week.

Question. Sir, may I just enlarge a little about jaundice? I don't know whether the surgeons here would agree with me, but laparotomy for obstructive jaundice usually turns out as either neoplasm or gallstone with complicating obstructive pancreatitis which is reflected in the biopsy which usually misses the causal carcinoma. Would the surgeons agree with this in their practice?

Pollock. Well, I agree that the biopsy reflecting chronic pancreatitis usually means that there is an obstructing neoplasm, but occasionally one does an operation and the patient lives for 10-15 years and in such a case one has to accept that the original diagnosis was true chronic stenosing pancreatitis without neoplasm.

QUESTION. I was very interested in Dr Howat's series of eight patients with steatorhoea and the notable lack of anaemia in this group and I wondered if after treatment any of this group did show an anaemia which would perhaps fit with the modern views of pancreas and iron absorption?

Howat. I can't answer this.

Dellipiani. A small point for Dr Howat, in his eight patients with steatorrhoea, I noticed that three of them had metabolic bone disease, and my impression was that this is an unusual complication of pancreatic steatorrhoea, and I wondered if he could tell me if this figure is correct. Is there a similar incidence in larger series?

Howat. These eight patients that I've shown, I have only shown spontaneous cases and do not claim that these represent the total number of cases of steatorrhoea in my whole group, but it so happens that metabolic bone disease is found in three. I think that the more you look for metabolic bone disease in chronic pancreatitis over a period of time the more often you will find it.

I followed these cases myself for most of eighteen years and this is why we picked out metabolic bone disease in these patients. One patient had a gastrectomy at one time and this may have been a contributory factor.

QUESTION. I should like to ask Dr Howat if he has any experience of the starch tolerance test that is done as a diagnostic test for chronic pancreatitis?

Howat. Dr Wolstenholme has had 1 years experience but we can't get any patients to take the starch! We have recently been producing a modified starch test which demonstrates severe pancreatic dysfunction, I suspect that it is not much use really from a diagnostic point of view-little or no use for diagnosing chronic pancreatitis. 